\title{
Novas perspectivas de fisioterapia respiratória em lesão medular - uma revisão sistemática
}

\author{
New perspectives for chest physical therapy in \\ spinal cord injury - a systematic review
}

Felipe Soares Macedo ${ }^{1}$

Clarissa Cardoso dos Santos Couto $\mathrm{Paz}^{2}$

Adson Ferreira da Rocha ${ }^{3}$

Cristiano Jacques Miosso ${ }^{3}$

Hellen Batista de Carvalho ${ }^{4}$

Sergio Ricardo Menezes Mateus ${ }^{2,3}$

\section{Descritores}

Tosse; Modalidade de fisioterapia;

Estimulação elétrica

\section{Keywords}

Cough; Physical therapy modalities;

Electric stimulation

\section{Submetido}

6 de Setembro de 2017

Aceito

30 de Outubro de 2017

\section{Resumo}

Objetivo: Descrever e analisar parâmetros e efeitos da estimulação elétrica de superfície na função muscular respiratória de pessoas com lesão medular, sobretudo durante a tosse.

Métodos: Foi realizada uma revisão sistemática da literatura, com base no Preferred Reporting Items for Systematic Reviews and MetaAnalyses. A busca foi realizada nas bases de dados PubMed, PEDro e LILACS, por meio dos seguintes descritores: "estimulação elétrica funcional", "eletroestimulação, estimulação elétrica", "tosse", "higiene brônquica", "quadriplegia", "lesão medular espinhal", "tetraplegia" e "sujeito com tetraplegia" - em espanhol, inglês e português, sem restrição quanto ao ano de publicação. Foram incluídos artigos com amostra de indivíduos com lesão medular assistidos por estimulação elétrica com desfecho relacionado ao sistema respiratório, e foram excluídos artigos com ensaios invasivos de estímulo a tosse.

Resultados: Os 12 artigos incluídos revelam heterogeneidade nos protocolos de eletroestimulação da função expiratória, que podem incluir frequências de 30 a $50 \mathrm{~Hz}$, com pulsos de 25 a 400 s, aplicada por até oito eletrodos distribuídos pelos músculos expiratórios e acessórios. 0 tempo de aplicação também foi variável e a amplitude de corrente frequentemente estimada pela percepção do paciente, podendo chegar a valores superiores a $100 \mathrm{~mA}$.

Conclusão: Apesar de não ser possivel estabelecer parâmetros rigorosos de fisioterapia por meio da estimulação elétrica, pela escassez e qualidade de estudos que comparem sistematicamente parâmetros de estimulação em subgrupos, foram observadas alterações positivas nas variáveis de função muscular respiratória avaliadas, como o pico de fluxo expiratório e de tosse, em pessoas com lesão medular cervical e torácica.

\begin{abstract}
Objective: To describe and analyze parameters and effects of surface electrical stimulation on the respiratory muscular function among individuals with spinal cord injuries, especially while coughing.

Methods: A systematic literature review was developed based on the Preferred Reporting Items for Systematic Reviews and Meta-Analyses. The search was conducted in the PubMed, PEDro, and LILACS databases, using the following descriptors: "functional electrical stimulation," "electrostimulation, electrical stimulation," "coughing," "bronchial hygiene," "quadriplegia," "spinal cord injury", "tetraplegia", and "individual with tetraplegia" - in Spanish, English and Portuguese, with no restrictions on year of publication. Inclusion criteria were: articles describing studies with samples of individuals with spinal cord injuries treated with electrical stimulation and outcomes related to the respiratory system. Articles containing studies with invasive cough stimulation trials were excluded.

Results: The 12 selected articles revealed the heterogeneity of electrostimulation protocols for expiratory function, which can include frequencies ranging from 30 to $50 \mathrm{~Hz}$; pulse from 25 to $400 \mu \mathrm{s}$; applied in up to eight electrodes distributed across the expiratory and accessory muscles. Time of administration also varied, and the current amplitude was usually estimated by the patient's perception, reaching values higher than $100 \mathrm{~mA}$.

Conclusion: Even though the review did not find rigorous parameters for physical therapy using electrical stimulation, because of the shortage and low-quality of the studies that systematically compare stimulation parameters among subgroups, positive changes were observed in the assessed respiratory muscle function variables, such as peak expiratory and cough flow in individuals with cervical and thoracic spinal cord injury.
\end{abstract}

${ }^{1}$ Faculdade de Medicina, Universidade de Brasília, Brasília, DF, Brasil.

Faculdade de Ceilândia, Universidade de Brasília, Brasília, DF, Brasil.

${ }^{3}$ Faculdade Gama, Universidade de Brasília, Brasília, DF, Brasil.

${ }^{4}$ Instituto de Medicina Integral Professor Fernando Figueira, Recife, PE, Brasil.

Conflitos de interesse: não há conflitos de interesse a declarar. 


\section{Introdução}

Historicamente, a lesão medular está associada a preocupantes taxas de incidência e prevalência. Nos Estados Unidos, por exemplo, ocorrem aproximadamente 11.000 novos casos por ano, e atualmente o país tem um contingente de até 288.000 pessoas vivendo com lesão medular espinal. Nesses casos, destacam-se, pela frequência, pelas sequelas e pelo ônus ao paciente e ao sistema de saúde, aqueles com lesóes causadoras de tetraplegias completas. ${ }^{(1)}$ No caso do Brasil, por outro lado, dados como esses ainda são desconhecidos, devido à ausência de um sistema eficaz de notificação, apesar das altas taxas de acidentes automobilísticos, que constituem a principal causa de lesão medular.

Além do desafiador impacto psíquico, social e econômico, a lesão medular espinal é caracterizada como uma complexa disfunção clínica e funcional, desencadeada pelo dano ao tecido nervoso medular, frequentemente por mecanismos traumáticos, como fratura-luxação de corpos vertebrais. Estes mecanismos invariavelmente repercutem com ampla e heterogênea alteração fisiológica, em que se destacam os prejuízos à função neuromotora - como a incapacidade de locomoção em detrimento da paralisia completa de membros, além de ineficaz força muscular respiratória para ventilação voluntária. ${ }^{(1,2)}$ De acordo com dados do $\mathrm{Na}$ tional Spinal Cord Injury Statistical Center, mudanças nos últimos 40 anos dos paradigmas de assistência e reabilitação favoreceram a taxa de sobrevivência e de expectativa de vida das pessoas com lesão medular. No entanto, vale destacar que as complicaçóes do sistema respiratório ainda correspondem às principais causas de internaçóes, reinternaçóes e óbitos de pessoas com lesão medular, totalizando mais de $20 \%$ das causas de morte que ocorrem, sobretudo, nos primeiros 6 meses após a lesão. ${ }^{(2,3)}$

Esse cenário pode estar relacionado às alteraçóes mecânicas respiratórias, desencadeadas pelo aumento do tônus brônquico, que associado à fraqueza e/ou paralisia da musculatura respiratória culminam em distúrbio restritivo não parenquimatoso. ${ }^{(3)} \mathrm{A}$ paralisia dos músculos respiratórios produz prejuízos para a função pulmonar, que, devido à incapacidade de gerar contraçóes musculares eficazes, afetam o volume inspiratório, o pico de fluxo da tosse e a pressão expiratória máxima.
Isso torna a tosse ineficiente para higiene brônquica, sendo um fator de risco para a atelectasia, para a infecção respiratória e para a insuficiência respiratória, instalando o cenário ideal para vulnerabilidade à internaçóes e óbito. ${ }^{(4-8)}$

Nesse contexto, intervençóes terapêuticas são indispensáveis para auxiliar a mecânica muscular respiratória e restabelecer funçóes diretamente relacionadas à eficiência da tosse, bem como para manter vias áreas pérvias e promover a higiene brônquica. ${ }^{(9-11)}$ Dentre algumas possibilidades de tratamento, condutas e recursos fisioterapêuticos têm se destacado pela capacidade de promover contraçóes musculares artificiais a partir da aplicação de estímulos elétricos de superfície nos músculos respiratórios. A modulação de parâmetros de estimulação elétrica pode gerar pressão intermitente nos músculos respiratórios paralisados, que, a partir disso, pode contribuir com o aumento da pressão intra-abdominal e repercutir no pico de fluxo de tosse, semelhantemente ao mecanismo fisiológico de higiene brônquica. ${ }^{(12-13)}$

Em consonância com a problemática exposta, o objetivo desta revisão sistemática da literatura (RSL) consiste em identificar e descrever parâmetros e efeitos da estimulação elétrica de superfície na função muscular respiratória, sobretudo durante a tosse, de indivíduos com lesão medular espinal.

\section{Métodos}

Foi realizada uma revisão sistemática da literatura (RSL) baseada no Preferred Reporting Items for Systematic Reviews and Meta-Analyses (PRISMA), organizada e conduzida de forma independente por dois pesquisadores experientes no tema - disfunção muscular respiratória (tosse voluntária), estimulação elétrica de superfície e lesão medular espinal. ${ }^{(14)}$

\section{Busca e identificação dos artigos}

A busca eletrônica foi realizada por dois pesquisadores com experiência no tema, de forma independente, havendo um terceiro avaliador à disposição em caso de divergências entre os resultados. Essa etapa abrangeu a biblioteca da Literatura Latino-Americana e do Caribe em Ciências da Saúde (LILACS), e bases de dados como 
Medical Literature Analysis and Retrieval Sistem Online (MedLine/PubMed) e Physiotherapy Evidence Database (PEDRo). Sendo propostos descritores em Espanhol, Inglês e Português, a princípio de acordo com a lista de Descritores em Ciências da Saúde (DeCS), e por seguinte foram acrescentadas outras palavras-chave frequentemente encontrados em artigos da temática.

Foram utilizados como descritores na língua portuguesa as expressões "estimulação elétrica funcional", "eletroestimulação", "estimulação elétrica”, "tosse", "higiene brônquica", "quadriplegia", "lesão medular espinhal", "tetraplegia" e "tetraplégico". No idioma inglês foram contemplados os termos "functional electric stimulation", "electrostimulation", "electrical stimulation", "cough", "mucociliary clearance”, "quadriplegia”, "spinal cord injury", "tetraplegia" e "tetraplegic". Para pesquisas publicadas em espanhol, foram atribuídas as palavras-chave "estimulación eléctrica funcional", "electroestimulación, estimulación eléctrica", "tos", "higiene bronquial", "cuadriplejía", "traumatismos de la médula espinal", "tetraplejía" e "tetrapléjico".

A equação de busca foi elaborada, a partir da combinação dos operadores $O R$ e $A N D$, conforme as características de cada base de dado. $\mathrm{Na}$ base $\mathrm{Pu}$ bmed e na biblioteca LILACS a pesquisa foi realizada no campo de "pesquisa avançada" (do inglês advanced search). Enquanto na base PEDro a busca foi realizada por meio do campo "busca simples" (do inglês simple search), "forma básica". A busca foi realizada no período de maio a dezembro de 2016 e atualizada em agosto de 2017. Além da busca eletrônica, foi realizada uma busca manual a partir das referências dos artigos encontrados nas bases eletrônicas, também foi realizada uma ampla busca na BCE/UnB - Biblioteca Central da Universidade de Brasília, a partir de periódicos impressos, trabalhos de conclusão de curso, dissertaçôes e teses.

\section{Seleção das evidências}

Para atingir o objetivo proposto, foi elaborada uma pergunta com base na estratégia (Patient Intervetion Comparison Outcome) PICO: Quais parâmetros e efeitos da estimulação elétrica de superfície utilizados para assistência fisioterapêutica de indivíduos com lesão medular?

Os artigos encontrados na busca eletrônica e manual foram previamente analisados pelo título e resu- mo. Como na etapa de busca, está análise também foi conduzida por dois pesquisadores com experiência no tema, de forma independente, havendo um terceiro avaliador à disposição em caso de divergências entre os relatórios de busca. Os artigos encontrados nas buscas eletrônica e manual foram confrontados, com o objetivo de identificar possíveis duplicidades intra e entre as bases de dados, como entre as estratégias de busca.

\section{Critérios de inclusão e exclusão}

Foram encaminhados para a sumarização de dados, por meio da leitura e análise plena dos artigos, em um quadro previamente estruturado (Quadro 1), evidências com amostra composta por humanos com lesão medular espinal, publicadas nos idiomas inglês, português e espanhol, cujas variáveis de intervenção correspondessem a estimulação elétrica de superfície e as variáveis de desfecho clínico fossem atribuídas à função respiratória, como, pico de fluxo expiratório e tosse, capacidade vital e volume corrente. No entanto, os artigos encontrados que não estavam em consonância com esses critérios foram eliminados. Dentre estes, foram excluídos estudos de revisão bibliográfica, revisão sistemática e semelhantes, além de artigos não indexados ou indisponíveis na íntegra em biblioteca, bases, e plataformas de dados pesquisadas. Também foram excluídos estudos com amostra com indivíduos sob ventilação mecânica, ventilação não-invasiva, submetidos a quaisquer intervençôes invasivas de estímulo à tosse e cujas variáveis de desfecho não representassem a função muscular respiratória ou pulmonar.

\section{Sumarização dos dados}

A sumarização dos dados contemplou a extração dos dados a seguir: autores e ano de publicação das evidências, características da amostra, instrumentos e/ou equipamentos de avaliação dos efeitos da estimulação elétrica de superfície na função muscular respiratória, e por fim, os desfechos pertinentes à intervenção terapêutica da estimulação elétrica na função respiratória da amostra de pessoas com lesão medular avaliada.

\section{Avaliação da qualidade metodológica}

Em decorrência da trajetória metodológica dos artigos incluídos, o rigor metodológico foi realizado por dois instrumentos distintos. No caso de ensaio clínico alea- 
Quadro 1. Evidências selecionadas com descrição dos parâmetros e efeitos da estimulação elétrica de superfície para estímulo da tosse em pessoas com lesão medular espinal

\begin{tabular}{|c|c|c|c|c|}
\hline Estudo & Amostra & Variáveis respiratórias avaliadas & Parâmetros de eletroestimulação & Desfechos na função respiratória \\
\hline Linder S H., (1993) ${ }^{(17}$ & $\begin{array}{l}8 \text { sujeitos do sexo } \\
\text { masculino } \\
\text { Classificação: } \\
\text { traumática e completa } \\
\text { Nivel: } C 4 \text { - } 5\end{array}$ & $\begin{array}{l}\text { Variáveis de função pulmonar e } \\
\text { pressão expiratória máxima (PEMax) } \\
\text { basais e com estimulação elétrica na } \\
\text { posição supina. }\end{array}$ & $\begin{array}{l}\text { Modalidade: Estimulação elétrica funcional (FES) } \\
\text { Frequência: } 50 \mathrm{~Hz} \\
\text { Largura do Pulso: } 300 \text { } \mu \mathrm{s} \\
\text { Duração do pulso: } 2-3 \mathrm{~s} \\
\text { Amplitude: } 100 \% \\
\text { Tempo on/off: - } \\
\text { Subida: } 0 \mathrm{~s} \\
\text { Onda: assimétrica bifásica } \\
\text { Tempo: Até } 30 \text { min } \\
\text { Eletrodos: } 4 \text { - } 8 \text { eletrodos na parede abdominal }\end{array}$ & $\begin{array}{l}\text { Aumento médio de } 33 \mathrm{~cm} \text { H2O na PEMax quando } \\
\text { aplicado eletroestimulação em relação aos valores } \\
\text { basais. Não houve diferença em reação ao uso de } \\
\text { cinta e da posição do sujeito. }\end{array}$ \\
\hline Jaeger R J. et al., (1993)(18) & $\begin{array}{l}14 \text { sujeitos do sexo } \\
\text { masculino e } 5 \text { do sexo } \\
\text { feminino Classificação: } \\
\text { completa e incompleta } \\
\text { Nivel: } C 4 \text { - } 7\end{array}$ & \begin{tabular}{|l|} 
Pico de fluxo expiratório (PFE) basal, \\
manual e durante a eletroestimulação.
\end{tabular} & 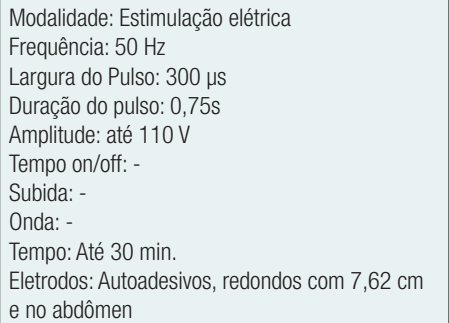 & $\begin{array}{l}\text { A eletroestimulação aumentou significativamente } \\
0 \text { PFE, atingindo o máximo de } 230 \mathrm{~L} \text { min } \pm 64 \\
\text { L/min, no entanto apresentou valores inferiores à } \\
\text { tosse manualmente assistida. }\end{array}$ \\
\hline Sorli J. et al., (1996) ${ }^{(19)}$ & $\begin{array}{l}1 \text { sujeito do sexo } \\
\text { feminino com LM } \\
\text { Classificação: } \\
\text { traumática e completa } \\
\text { Nivel: } C 6 \text { - } 7\end{array}$ & $\begin{array}{l}\text { Volume do tronco (peito e no tórax) } \\
\text { por sistema de rastreamento óptico, } \\
\text { aferido na posição supina antes e } \\
\text { durante a eletroestimulação. }\end{array}$ & 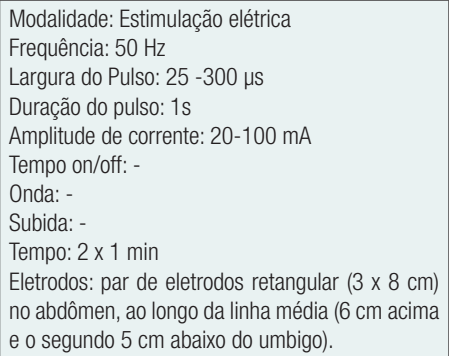 & $\begin{array}{l}0 \text { volume do tórax variou com a eletroestimulação } \\
\text { que pode sugerir aumento do volume corrente. }\end{array}$ \\
\hline Zupan A. et al., (1997) 20 & $\begin{array}{l}14 \text { sujeitos (11 do sexo } \\
\text { masculino e } 3 \text { do sexo } \\
\text { feminino) } \\
\text { Classificação: completa } \\
\text { e incompleta } \\
\text { Nivel: C4 - } 7\end{array}$ & $\begin{array}{l}\text { Capacidade vital forçada (CVF) e } \\
\text { volume expiratório forçado no primeiro } \\
\text { segundo }\left(\text { VEF } F_{1} \text { ) avaliados antes e após }\right. \\
0 \text { protocolo na posição sentada e } \\
\text { supina. E eletroestimulação associada } \\
\text { ao treinamento muscular foi aplicado } \\
\text { por um fisioterapeuta e pelo paciente. }\end{array}$ & $\begin{array}{l}\text { Modalidade: Estimulação elétrica } \\
\text { Frequência: } 50 \mathrm{~Hz} \\
\text { Largura do Pulso: } 300 \mu \mathrm{s} \\
\text { Duração do pulso: } 0,75 \mathrm{~s} \\
\text { Amplitude de voltagem: até } 110 \mathrm{~V} \\
\text { Tempo on/off: - } \\
\text { Onda: - } \\
\text { Subida: - } \\
\text { Tempo: } 20 \text { - } 30 \text { min. } \\
\text { Eletrodos: } 4 \text { eletrodos no abdômen }\end{array}$ & $\begin{array}{l}\text { Aumento médio de } 19 \% \text { na CV na posição } \\
\text { sentada e } 17,5 \% \text { na posição supina. Aumento } \\
\text { médio no VEF }{ }_{1} 20,5 \% \text { na posição sentada e } 16 \% \\
\text { na posição deitada. Após um mês sem aplicação } \\
\text { do protocolo o aumento médio foi de } 9,5 \% \text { da CVF } \\
\text { e } 7 \% \text { de VEF }\end{array}$ \\
\hline Cheng et al., $(2006)^{21}$ & $\begin{array}{l}\text { Grupo ensaio: } 13 \\
\text { sujeitos (11 do sexo } \\
\text { masculino e } 2 \text { do sexo } \\
\text { feminino) } \\
\text { Nível: } C 4 \text { - } 7 \\
\text { Classificação: completa } \\
\text { Grupo controle: } 13 \\
\text { sujeitos (10 do sexo } \\
\text { masculino e } 3 \text { do sexo } \\
\text { feminino) } \\
\text { Nível: } C 4 \text { - } 7\end{array}$ & $\begin{array}{l}\text { Variáveis de função pulmonar aferidos } \\
\text { na posiçãa supina antes e após (3 } \\
\text { semanas, } 3 \text { meses e } 6 \text { meses) cada } \\
\text { protocolo (protocolo de fisioterapia } \\
\text { versus protocolo de fisioterapia } \\
\text { associada a estimulação). }\end{array}$ & $\begin{array}{l}\text { Modalidade: Estimulação elétrica neuromuscular } \\
\text { Frequência: } 30 \mathrm{~Hz} \\
\text { Largura do Pulso: } 300 \mu \mathrm{s} \\
\text { Duração do Pulso: - } \\
\text { Amplitude de corrente: } 0 \text { - } 100 \mathrm{~mA} \\
\text { Tempo on/off: } 4 \mathrm{~s} / 4 \mathrm{~s} \\
\text { Onda: onda bifásica simétrica } \\
\text { Subida: } 0,5 \mathrm{~s} \\
\text { Tempo: } 30 \text { min } \\
\text { Eletrodos: dispostos nos pontos motores da } \\
\text { porção clavicular do peitoral bilateral e nos } \\
\text { músculos abdominais ( } 3 \mathrm{~cm} \text { acima do umbigo) }\end{array}$ & $\begin{array}{l}\text { As variáveis de função pulmonar, como } \\
\text { capacidade vital, capacidade vital forçada, } \\
\text { volume expiratório forçado em } 1 \text { segundo e } \\
\text { pico de fluxo melhoraram após a intervenção } \\
\text { fisioterapêutica, no entanto estes dados foram } \\
\text { mais favoráveis quando a conduta fisioterapêutica } \\
\text { foi atrelada a eletroestimulação. Após } 6 \text { meses } \\
\text { concluiu que } 6 \text { dos } 13 \text { indivíduos do grupo } \\
\text { controle apresentaram complicações pulmonares, } \\
\text { enquanto no grupo experimental apenas } 1 \text {. Ainda } \\
\text { no grupo tratado apenas com fisioterapia um } \\
\text { sujeito foi à traqueostomia e ventilação invasiva. }\end{array}$ \\
\hline Golle et al., (2006) ${ }^{122}$ & $\begin{array}{l}4 \text { sujeitos ( } 3 \text { do sexo } \\
\text { masculino e } 1 \text { do sexo } \\
\text { feminino) } \\
\text { Classificação: completa }\end{array}$ & $\begin{array}{l}\text { Pico de fluxo de tosse (PFT) e } \\
\text { volume corrente (VC) aferidos } \\
\text { na posição sentada ou supina, } \\
\text { durante a respiração tranquila e a } \\
\text { tosse estimuladas por meio de um } \\
\text { eletroestimulador controlado por um } \\
\text { algoritmo de identificação de fluxo } \\
\text { respiratório. }\end{array}$ & $\begin{array}{l}\text { Modalidade: Estimulação elétrica } \\
\text { Frequência: } 50 \mathrm{~Hz} \\
\text { Largura do Pulso: } 100 \text { - } 400 \mu \mathrm{s} \\
\text { Duração do Pulso: - } \\
\text { Amplitude de corrente: } 30-100 \mathrm{~mA} \\
\text { Tempo on/off: - } \\
\text { Onda: Monofásica equilibrada } \\
\text { Subida: - } \\
\text { Tempo: - } \\
\text { Eletrodos: } 4 \text { no reto abdominal, } 2 \text { no transverso } \\
\text { abdominal (retangulares de } 33 \mathrm{~mm} \text { x } 53 \mathrm{~mm} \text { ), } 2 \\
\text { no externo e } 2 \text { no interno obliquo (redondos com } \\
50 \mathrm{~mm}\end{array}$ & $\begin{array}{l}\text { A estimulação elétrica automatizada aumentou em } \\
\text { até } 71 \% \text { o VC e } 54 \% \text { o PFT com a estimulação. } \\
0 \text { volume apresentou a melhor variação de } 0,35 \\
\text { L para } 0,6 \mathrm{~L} \text { e a menor diferença de } 0,32 \mathrm{~L} \text { para } \\
0,35 \mathrm{~L} \text { respectivamente. Enquanto a melhor taxa } \\
\text { de variação da tosse foi de } 2,2 \mathrm{~L} / \mathrm{s} \text { para } 3,4 \mathrm{~L} / \mathrm{s} \\
\text { com estimulação. }\end{array}$ \\
\hline
\end{tabular}


continuação

\begin{tabular}{|c|c|c|c|c|}
\hline Estudo & Amostra & Variáveis respiratórias avaliadas & Parâmetros de eletroestimulação & Desfechos na função respiratória \\
\hline Spivak et al., $(2007)^{(23}$ & $\begin{array}{l}10 \text { sujeitos do sexo } \\
\text { masculino } \\
\text { Nível: } \text { C4 - } 7 \\
\text { Classificação: } \\
\text { completa. }\end{array}$ & $\begin{array}{l}\text { Variáveis de função pulmonar } \\
\text { avaliadas: sem assistência, } \\
\text { com fisioterapia manual, com } \\
\text { eletroestimulação pelo cuidador, com } \\
\text { eletroestimulação pelo paciente e } \\
\text { estimulação ativada por sinais de } \\
\text { EMG aferidos na posição supina. }\end{array}$ & $\begin{array}{l}\text { Modalidade: Estimulação elétrica funcional } \\
\text { Frequência: } 50 \mathrm{~Hz} \\
\text { Largura do Pulso: } 300 \text { us } \\
\text { Duração do pulso: - } \\
\text { Amplitude de corrente: } 0 \text { - } 100 \mathrm{~mA} \\
\text { Tempo on/off: - } \\
\text { Onda: - } \\
\text { Subida: - } \\
\text { Tempo: - } \\
\text { Eletrodos: } 4 \text { eletrodos à esquerda e } 4 \text { à direita } \\
\text { do abdôm }\end{array}$ & $\begin{array}{l}0 \text { pico de fluxo expiratório, a capacidade vital } \\
\text { forçada e a ventilação voluntária máxima } \\
\text { aumentaram seus valores em todas as forças } \\
\text { de assistências, exceto na eletroestimulação } \\
\text { autoaplicada pelo paciente. A fisioterapia manual } \\
\text { apresentou } 0 \text { melhor desempenho quando } \\
\text { comparada as outras formas de assistência a } \\
\text { tosse. }\end{array}$ \\
\hline Golle et al., (2008) ${ }^{(24}$ & $\begin{array}{l}4 \text { sujeitos ( } 3 \text { do sexo } \\
\text { masculino e } 1 \text { do sexo } \\
\text { feminino) } \\
\text { Nível: } C 4 \text { - } 6 \\
\text { Classificação: } \\
\text { completa }\end{array}$ & $\begin{array}{l}\text { Pico de fluxo de tosse (PFT), volume } \\
\text { corrente (VC), volume de reserva } \\
\text { expiratório (VRE), ventilação minuto } \\
\text { (VM), frequência respiratória e } \\
\text { pressão parcial de } \mathrm{CO}_{2} \text { no ar expirado } \\
\text { aferidos na posição sentada durante } \\
\text { a respiração tranquila e tosse } \\
\text { eletroestimulada automaticamente } \\
\text { por meio de um controlador de fluxo } \\
\text { respiratório. }\end{array}$ & $\begin{array}{l}\text { Modalidade:Estimulação elétrica funcional } \\
\text { Frequência: } 50 \mathrm{~Hz} \\
\text { Largura do Pulso: } 400 \text { } \mu \mathrm{s} \\
\text { Duração do pulso: - } \\
\text { Amplitude de corrente: } 30 \text { - } 100 \mathrm{~mA} \\
\text { Tempo on/off: - } \\
\text { Onda: - } \\
\text { Subida: - } \\
\text { Tempo: - } \\
\text { Eletrodos: } 8 \text { eletrodos autoadesivos distribuídos } \\
\text { em } 2 \text { pares no músculo reto abdominal, } 1 \text { par no } \\
\text { músculo transverso abdominal e } 1 \text { no músculo } \\
\text { externo e } 1 \text { no interno obliquo abdominal }\end{array}$ & $\begin{array}{l}\text { Foi observado aumento de até } 50 \% \text { no número de } \\
\text { tosses durante a eletroestimulação, com aumento } \\
\text { do PFT em todos os sujeitos, sendo o aumento } \\
\text { máximo de } 0,49 \mathrm{~L} / \mathrm{s}(\mathrm{p}<0.05) \text {. VC variou até + } \\
0,23 \mathrm{~L} \text {. A capacidade vital forçada melhorou até + } \\
0,49 \mathrm{~L} / \mathrm{s} \text {. E o PFT melhorou em todos os sujeitos } \\
\text { avaliados. O VC também aumento em todos } \\
\text { os participantes. As demais variáveis avaliadas } \\
\text { apresentaram dados heterogêneos. }\end{array}$ \\
\hline Butler et al., (2010)(25) & $\begin{array}{l}11 \text { sujeitos (8 do sexo } \\
\text { masculino e } 3 \text { do sexo } \\
\text { feminino) } \\
\text { Nível: C3 - T6 } \\
\text { Classificação: } \\
\text { completa e incompleta }\end{array}$ & $\begin{array}{lrr}\text { Capacidade } & \text { inspiratória } & (\mathrm{Cl}), \\
\text { capacidade vital } & \text { (CV), capacidade } \\
\text { vital forçada (CVF), volume expiratório } \\
\text { forçado em } 1 \text { segundo }\left(\mathrm{VEF}_{1} \text { ) aferidos }\right. \\
\text { na posição sentada durante a tosse } \\
\text { com a glote ocluída } & \text { com er sem } \\
\text { eletroestimulação. } & \text { A } & \text { estimulação } \\
\text { foi controlada } & \text { e } & \text { disparada } \\
\text { automaticamente em } & 6 & \text { sujeitos e } \\
\text { autocontrolada por } 5 . & \end{array}$ & $\begin{array}{l}\text { Modalidade: Estimulação elétrica funcional } \\
\text { Frequência: } 50 \mathrm{~Hz} \\
\text { Largura do Pulso: - } \\
\text { Duração do pulso: } 1 \mathrm{~s} \\
\text { Amplitude de corrente } 50-350 \mathrm{~mA} \\
\text { Tempo on/off: - } \\
\text { Onda: - } \\
\text { Subida: - } \\
\text { Tempo: } 3 \text { aplicações } \\
\text { Eletrodos: } 2 \text { pares de eletrodos }(4 \times 18 \mathrm{~cm}) \\
\text { bilateralmente localizados na porção latero- } \\
\text { posterior do tronco }\end{array}$ & $\begin{array}{l}\text { As variáveis de função pulmonar aumentaram } \\
\text { nos indivíduos submetidos a estimulação } \\
\text { elétrica durante a tosse. } 0 \text { pico de fluxo de tosse } \\
\text { aumentou em } 36 \% \text {, enquanto o fluxo expiratório } \\
\text { médio } 80 \% \text { e volume pulmonar expirado } 41 \% \text {. A } \\
\text { oclusão da glote durante as manobras também } \\
\text { contribuiu para melhoras as variáveis de função } \\
\text { pulmonar, no entanto, os melhores resultados } \\
\text { foram evidenciados quando a eletroestimulação foi } \\
\text { aplicada automaticamente pelo sistema. }\end{array}$ \\
\hline McLachlan et al., (2013) ${ }^{(12)}$ & $\begin{array}{l}12 \text { sujeitos (11 do } \\
\text { sexo masculino e } 1 \text { do } \\
\text { sexo feminino) } \\
\text { Nível: C3 - } 6 \\
\text { Classificação: } \\
\text { completa e incompleta }\end{array}$ & $\begin{array}{l}\text { Capacidade vital forçada (FVC), } \\
\text { volume exalado forçado em } 1 \text { segundo } \\
\text { (VEF1), pico de fluxo expiratório } \\
\text { (PFE), pressão expirada máxima } \\
\text { (PEMax), capacidade vital forçada } \\
\text { (CVF), volume exalado forçado em } \\
1 \text { segundo (VEF1), aferidos durante } \\
\text { a tosse voluntária antes, durante e } \\
\text { após um programa de reabilitação } \\
\text { muscular respiratória por meio de } \\
\text { estimulação elétrica funcional. }\end{array}$ & $\begin{array}{l}\text { Modalidade: Estimulação elétrica funcional } \\
\text { Frequência: } 30 \mathrm{~Hz} \\
\text { Largura do Pulso: } 50 \mu \mathrm{s} \\
\text { Duração do pulso: - } \\
\text { Amplitude de corrente: } 0 \text {-120 mA } \\
\text { Tempo on/off: - } \\
\text { Onda: Bifásica } \\
\text { Subida: - } \\
\text { Tempo: } 6 \text { semanas, } 5 \text { dias por semana, } 11 \text { - } 230 \\
\text { min } \\
\text { Eletrodos: } 8 \text { eletrodos autoadesivos distribuídos } \\
\text { em } 2 \text { pares no músculo oblíquo abdominal } \\
\text { (bilateral) e } 2 \text { pares no músculo reto abdominal }\end{array}$ & $\begin{array}{l}\text { A estimulação não produziu contrações } \\
\text { vigoras em todos os sujeitos, como também } \\
\text { não foi suportada por todo grupo avaliado. As } \\
\text { repercussões do protocolo na função pulmonar a } \\
\text { foram heterogêneas, sendo encontradas discretas } \\
\text { alterações positivas no pico de fluxo de tosse, } \\
\text { na pressão expiratória máxima e no volume } \\
\text { corrente durante o treinamento. No entanto, após } \\
\text { o treinamento não foi encontrada significância } \\
\text { estatística no FEV1, na relação VEF1 / CVF e no } \\
\text { PFE. }\end{array}$ \\
\hline McBain et al., (2013) & $\begin{array}{l}\text { Grupo A: } 7 \text { sujeitos (11 } \\
\text { do sexo masculino } \\
\text { Nível: C4 - T5 } \\
\text { Classificação: } \\
\text { completa e incompleta } \\
\text { Grupo B: } 8 \text { sujeitos (do } \\
\text { sexo masculino } \\
\text { Nível: C4 - } 7 \\
\text { Classificação: } \\
\text { completa }\end{array}$ & $\begin{array}{l}\text { Capacidade inspiratória (Cl), } \\
\text { capacidade vital (CV), capacidade } \\
\text { vital forçada (CVF), volume expiratório } \\
\text { forçado em } 1 \text { segundo (VEF1), pico } \\
\text { de fluxo expiratório (PFE) aferidos na } \\
\text { posição sentado durante o estado } \\
\text { basal, a tosse voluntária, e na fase } \\
\text { expulsiva da tosse ambas submetidas } \\
\text { a estímulos elétricos. }\end{array}$ & $\begin{array}{l}\text { Modalidade: Estimulação elétrica funcional } \\
\text { Frequência: } 50 \mathrm{~Hz} \\
\text { Largura do Pulso: - } \\
\text { Duração do pulso: } 3 \mathrm{~s} \\
\text { Amplitude de corrente: até } 250 \mathrm{~mA} \\
\text { Tempo on/off: } \\
\text { ubida: - } \\
\text { STempo: } 6 \text { semanas, } 5 \text { dias por semana (5 séries } \\
\text { de } 10 \text { tosses por dia) } \\
\text { Eletrodos: } 2 \text { eletrodos ( } 5 \text { x } 18 \mathrm{~cm} \text { ) bilateralmente } \\
\text { localizados na porção latero-posterior do } \\
\text { abdômen }\end{array}$ & $\begin{array}{l}\text { A estimulação melhorou a tosse aguda nos } \\
\text { pacientes com lesão medular alta. Durante a tosse } \\
\text { voluntária, a estimulação da tosse aumentou de } \\
50 \% \text { no PFE. Após } 6 \text { semanas de treinamento da } \\
\text { tosse, houve aumento significativo do PEF }(3,1 \pm \\
0,1 \text { a } 3,6 \pm 0,1 \mathrm{~L} / \mathrm{s}) \text { e do fluxo expiratório da tosse } \\
\text { durante a tosse voluntária não estimulada, nos } \\
\text { sujeitos avaliados. As medidas da função pulmonar } \\
\text { melhoraram após o treinamento. } 0 \text { FEV1 aumentou } \\
\text { de } 1,3 \pm 0,1 \text { para } 1,4 \pm 0,1 \mathrm{~L}(\mathrm{P}=0,002) \text {, o FVC } \\
\text { aumentou de } 1,7 \pm 0,2 \text { para } 1,9 \pm 0,2 \mathrm{~L}(\mathrm{P}= \\
0,03) \text {, o } \mathrm{PEF} \text { aumentou de } 2,1 \pm 0,1 \text { para } 2,4 \pm \\
0,2 \mathrm{~L} / \mathrm{s}(\mathrm{P}=0,03), 0 \mathrm{VC} \text { aumentou de } 1,5 \pm 0,1 \\
\text { para } 1,8 \pm 0,1 \mathrm{~L}(\mathrm{P}=0,009) \text { e } \mathrm{IC} \text { aumentou de } 1,4 \\
\pm 0,1 \text { para } 1,6 \pm 0,1 \mathrm{~L}(\mathrm{P}=0,04) \text {. }\end{array}$ \\
\hline
\end{tabular}


continuação

\begin{tabular}{|c|c|c|c|c|}
\hline Estudo & Amostra & Variáveis respiratórias avaliadas & Parâmetros de eletroestimulação & Desfechos na função respiratória \\
\hline McBain et al., (2015) ${ }^{127}$ & \begin{tabular}{|l|}
7 sujeitos \\
Nível: C4 - 7 \\
Classificação: completa \\
e incompleta
\end{tabular} & $\begin{array}{l}\text { Variáveis de função pulmonar } \\
\text { aferidos na posição sentada durante } \\
\text { o estado basal, a tosse voluntária, } \\
\text { e na fase expulsiva da tosse ambas } \\
\text { submetidas a estímulos elétricos. }\end{array}$ & $\begin{array}{l}\text { Modalidade: Estimulação elétrica funcional } \\
\text { Frequência: } 50 \mathrm{~Hz} \\
\text { Largura do Pulso: - } \\
\text { Duração do pulso: } 1 \mathrm{~s} \\
\text { Amplitude de corrente } 50 \text { - } 360 \mathrm{~mA} \\
\text { Tempo on/off: - } \\
\text { Onda: - } \\
\text { Subida: - } \\
\text { Tempo: - } \\
\text { Eletrodos: } 2 \text { pares de eletrodos }(4 \times 18 \mathrm{~cm}) \\
\text { bilateralmente localizados na porção latero- } \\
\text { posterior do abdômen }\end{array}$ & $\begin{array}{l}0 \text { maior percentual de pacientes submetidos à } \\
\text { estimulação elétrica durante a tosse aumentaram } \\
\text { o fluxo tosse simultaneamente ao aumento da } \\
\text { amplitude da corrente aplicada. } 0 \text { pico de fluxo } \\
\text { de tosse atingiu } 4,0 \pm 0,4 \mathrm{~L} / \mathrm{s} \text { com parâmetros } \\
\text { de } 80 \text { a } 240 \mathrm{~mA} \text { e foi significativamente maior } \\
\text { do que na tosse voluntária sem eletroestimulação } \\
\text { que apresentou } 2,2 \pm 0,1 \mathrm{~L} / \mathrm{s}, \mathrm{p}<0,05 \text {. } 0 \\
\text { volume expiratório total também foi maior durante } \\
\text { a estimulação atingindo } 2,1 \mathrm{~L} \text { enquanto sem } \\
\text { estimulação } 1,3 \text { com os mesmos parâmetros. }\end{array}$ \\
\hline
\end{tabular}

LME - Lesão medular espinal; C - a nível de lesão cervical; Pressão expiratória máxima - PEMax; Não informado (-); Pico de fluxo expiratório - PFE; FES - Estimulação elétrica funcional (do inglês Functional Electrical Stimulation); $\mathrm{Hz}$ - abreviação de hertz, unidade de medida equivalente a um ciclo por segundo; $\mu$ - símbolo de mícron, unidade de comprimento que equivale a $1 / 1000000$ de um metro; $s$ - abreviação de segundos, unidade de medida de tempo; $\mathrm{mA}$ - abreviação de miliampères, unidade de medida de corrente elétrica que equivale a um milésimo de ampère; Min - abreviação de minutos, unidade de medida de tempo, correspondente a 60 segundos. V - abreviação de volts, unidade de medida de diferença de potencial.

tório foi utilizada a escala de avaliaçáo da Physiotherapy Evidence Database (PEDro). ${ }^{(15)}$ Enquanto em artigos de estudos observacionais foi aplicado o Strengthening the Reporting of Observational Studies in Epidemiology $(S T R O B E) .{ }^{(16)}$ A sumarização dos dados e avaliação metodológica também seguiram critérios de avaliação independente, sendo realizadas por dois pesquisadores com experiência no tema, com a disponibilizaçấo de um terceiro avaliar no caso de eventuais divergências. No entanto, em nenhuma das etapas descritas foi necessária a participaçấo do terceiro avaliador.

\section{Resultados}

A trajetória pertinente à busca, seleção, elegibilidade e inclusão das evidências científicas relacionadas aos efeitos e parâmetros da estimulação elétrica de superfície na função respiratória de pessoas com lesão medular pode ser visualizada no fluxograma da figura 1 .

Conforme as estratégias estabelecidas 74 arquivos foram encontrados na base PEDro, 44 no PubMed e nenhum nos demais, totalizando 118 achados. Enquanto a busca manual obteve 4 documentos pertinentes ao tema, sendo dois artigos publicados no período anterior a 1993. Posteriormente, foi investigada a existência de duplicidade entre os achados, sendo encontradas 22 duplicidades entre as bases. Ao final 100 artigos foram considerados supostamente relacionados à pergunta da pesquisa, no entanto 72 não foram selecionados em razão dos critérios de elegibilidade. A razão para essa exclusão foi o fato de que, apesar da estratégia de busca utilizada tentar selecionar documentos potencialmente elegíveis, muitas evidências não atendem aos critérios de inclusão. Por exemplo, artigos com ensaios realizados em animais foram eliminados em decorrência dessa revisão sistemática incluir pesquisas restritas à amostra composta por humanos. Enquanto das 28 evidências incluídas para avaliação plena, 16 foram excluídos em razão da incompatibilidade com os critérios de elegibilidade citados no fluxograma da figura 1. De forma detalhada, as razões de exclusão correspondem a estudos conduzidos em indivíduos sob ventilação mecânica invasiva ou ventilação nãoinvasiva. Foram também excluídos estudos realizados a partir de intervençôes invasivas de estímulo à tosse, como estimulação elétrica epidural, bem como artigos de revisão e ainda dois dos 28 artigos foram publicados no período anterior a 1993. No caso destes dois artigos, a Biblioteca Central da Universidade de Brasília solicitou, via e-mail, a versão completa, mas não foi obtida resposta. Além disso, um desses dois era uma carta ao autor, de forma que de qualquer forma seria excluído com base no critério anterior.

Concluído o processo de elegibilidade, 12 artigos foram incluídos para sumarização dos dados e avaliação da qualidade metodológica. Nesse sentido, as evidências extraídas contêm artigos no idioma inglês, publicados entre os anos de 1993 e 2015 e majoritariamente com desenho observacional. Estes artigos apresentam heterogênea amostra em relação ao nível (cervical e torácico) e classificação da lesão medular espinal, tempo de lesão, gênero e idade. Em relaçáo aos protocolos de avaliação e intervenção fisioterapêutica por meio de estimulaçáo elétrica de superfície também foi constatada variabilidade.

A função muscular respiratória foi aferida por medidas de função pulmonar, pelo pico de fluxo de tos- 


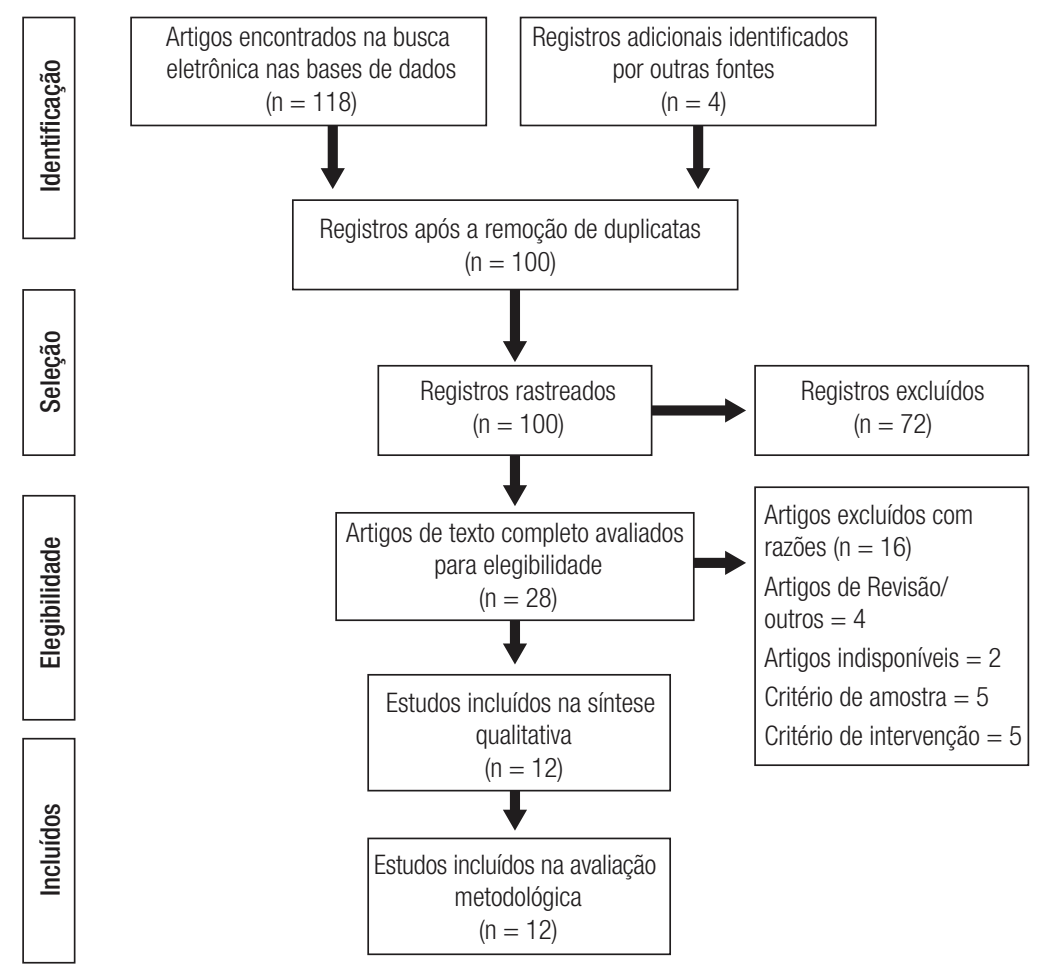

Figura 1. Fluxograma da seleção de evidências baseado nas diretrizes do PRISMA

Tabela 1. Avaliação do rigor metodológico pelo STROBE: estudos observacionais de estimulação elétrica para assistência a tosse em indivíduos com lesão medular espinal

\begin{tabular}{|c|c|c|c|c|c|c|c|c|c|c|c|c|c|c|c|c|c|c|c|c|c|c|c|}
\hline \multirow{2}{*}{ Artigo } & \multicolumn{22}{|c|}{ Itens $S T R O B E$} & \multirow{2}{*}{$\begin{array}{l}\text { Total de } \\
\text { Itens }\end{array}$} \\
\hline & 1 & 2 & 3 & 4 & 5 & 6 & 7 & 8 & 9 & 10 & 11 & 12 & 13 & 14 & 15 & 16 & 17 & 18 & 19 & 20 & 21 & 22 & \\
\hline Linder S H., (1993) ${ }^{(17)}$ & 0 & 1 & 1 & 1 & 0 & 1 & 0 & 1 & 0 & 0 & 1 & 0 & 1 & 0 & 1 & 0 & 0 & 1 & 0 & 1 & 1 & 0 & $11 / 22$ \\
\hline Jaeger R J. et al., (1993)(18) & 0 & 1 & 0 & 1 & 0 & 0 & 0 & 1 & 1 & 0 & 1 & 1 & 1 & 1 & 1 & 1 & 0 & 1 & 0 & 1 & 1 & 0 & $13 / 22$ \\
\hline Sorli J. et al., (1996) ${ }^{(19)}$ & 0 & 1 & 1 & 1 & 0 & 1 & 0 & 1 & 0 & 0 & 1 & 0 & 1 & 1 & 1 & 1 & 0 & 1 & 0 & 1 & 1 & 0 & $13 / 22$ \\
\hline Golle et al., $(2006)^{(22)}$ & 0 & 1 & 1 & 1 & 0 & 0 & 0 & 1 & 0 & 0 & 1 & 0 & 1 & 1 & 1 & 0 & 0 & 1 & 0 & 1 & 1 & 0 & $11 / 22$ \\
\hline Spivak et al., (2007) ${ }^{(23)}$ & 1 & 1 & 1 & 1 & 0 & 0 & 0 & 1 & 0 & 0 & 1 & 1 & 1 & 1 & 1 & 1 & 0 & 1 & 1 & 1 & 0 & 0 & $13 / 22$ \\
\hline Golle et al., (2008) ${ }^{(24)}$ & 0 & 1 & 1 & 1 & 1 & 1 & 0 & 1 & 0 & 0 & 1 & 1 & 1 & 1 & 1 & 0 & 0 & 1 & 1 & 1 & 0 & 0 & 1422 \\
\hline Butler et al., (2010) & 0 & 1 & 1 & 1 & 0 & 1 & 0 & 1 & 0 & 0 & 1 & 1 & 1 & 1 & 1 & 1 & 0 & 1 & 0 & 1 & 0 & 1 & $14 / 22$ \\
\hline McLachlan et al., (2013) & 1 & 1 & 1 & 1 & 1 & 1 & 0 & 1 & 0 & 0 & 1 & 1 & 1 & 1 & 1 & 1 & 0 & 1 & 1 & 1 & 0 & 0 & $16 / 22$ \\
\hline McBain et al., (2015) & 0 & 1 & 1 & 1 & 0 & 1 & 0 & 1 & 0 & 0 & 1 & 1 & 1 & 1 & 1 & 1 & 0 & 1 & 0 & 1 & 0 & 1 & $14 / 22$ \\
\hline
\end{tabular}

0 - Não apresenta 0 item preconizado; 1 - Apresenta o item preconizado; 0 percentual refere-se ao valor total obtido nos itens avaliados, sendo $100 \%$ correspondentes a 22 itens

Tabela 2. Avaliação do rigor metodológico pela escala de PEDro: ensaio clínico de estimulação elétrica na assistência a tosse em indivíduos com lesão medular espinal

\begin{tabular}{|c|c|c|c|c|c|c|c|c|c|c|c|c|}
\hline \multirow{2}{*}{ Artigo } & \multicolumn{11}{|c|}{ Itens da escala de PEDro } & \multirow{2}{*}{ Pontuação tota } \\
\hline & $1^{*}$ & 2 & 3 & 4 & 5 & 6 & 7 & 8 & 9 & 10 & 11 & \\
\hline Zupan A. et al., (1997) & * & 1 & 0 & 1 & 0 & 0 & 0 & 1 & 0 & 0 & 0 & $2 / 10$ \\
\hline Cheng et al., (2006)(21) & * & 1 & 0 & 1 & 0 & 0 & 0 & 1 & 0 & 1 & 1 & $5 / 10$ \\
\hline McBain et al., $(2013)^{(26)}$ & * & 1 & 0 & 1 & 0 & 0 & 0 & 0 & 0 & 0 & 0 & $2 / 10$ \\
\hline
\end{tabular}

0 - Não apresenta 0 critério preconizado; 1 - Apresenta o critério preconizado; *Critério não pontuado segundo as recomendações da escala PEDro

se ou expiratório e pela pressão expiratória máxima. Estas medidas permitem observar que o desempenho basal dos indivíduos avaliados apresentaram valores abaixo do previsto. Os parâmetros de eletroestimulação aplicados, detalhados no quadro 1, mostram que o recurso utilizado em diferentes modalidades e modulaçóes, com amplitude de corrente de até 360 miliampére $(\mathrm{mA})$, foi capaz de alterar positivamente as variáveis de função muscular respiratória avaliadas, como o pico de fluxo de tosse (Tabelas 1 e 2). 


\section{Discussão}

As evidências encontradas nesta revisão atestam que a intervenção fisioterapêutica, por meio da eletroestimulação de superfície, pode melhorar a função muscular respiratória dos indivíduos com diferentes níveis e complexidade de lesão medular espinal, com repercussões em curto, médio e longo prazo, inclusive na prevenção de complicações respiratórias graves, como a pneumonia. ${ }^{(9,12,17,21,27)}$

Em relação à amostra incluída nos ensaios, os estudos que mencionaram a idade dos pacientes demonstram panorama semelhante as estatísticas de centro especializados no acompanhamento de pessoas com lesão medular. A maioria dos acometidos por lesão medular está na faixa de idade da população economicamente ativa, nesta revisão os indivíduos apresentam idade entre 16 e 60 anos, com predominância do sexo masculino e com etiologia frequentemente relacionada a trauma. ${ }^{(17-27)}$ Essas características corroboram com pesquisas realizadas no Estados Unidos da América, em alguns países da Europa e no Brasil. ${ }^{(2,28,29,30)}$

As evidências avaliadas atestam que variáveis de função pulmonar, muscular respiratória e de tosse, como - capacidade vital (CV), volume expiratório forçado $\left(\mathrm{VEF}_{1}\right)$, pressão expiratória máxima (PEMax), pico de fluxo expiratório (PFE) e de tosse (PFT), utilizadas para classificação da amostra e avaliação dos protocolos de intervenção, apresentam valores abaixo do previsto, no entanto sem presença de morbidade parenquimatosa. ${ }^{(17-27)}$ Diante de perfis clínicos complexos, que incluem, além da atenção global à função musculoesquelética, cuidados com a função muscular respiratória e pulmonar as intervençóes por meio de estímulos elétricos em pacientes com lesão medular são classicamente conhecidas na arsenal de competências do profissional de fisioterapia. No entanto, a utilização de estímulos elétricos, como as condutas fisioterapêuticos para reabilitação foram modificados como mostram as evidências de assistência fisioterapêutica reunidos nos últimos 30 anos. ${ }^{(31-33)}$

Os protocolos de eletroestimulação de superfície à função pulmonar e à tosse incluem diferentes modalidades de corrente, sendo as mais citadas nos estudos primários avaliados, a estimulação elétrica funcional (FES, do inglês Functional Electrical Stimulation) e a estimulação neuromuscular funcional (NMES, do inglês NeuroMuscular Electrical Stimulation). ${ }^{(17-27)} \mathrm{Em}$ relação aos efeitos dos protocolos analisados constatou-se que diferentes modulaçóes de eletroestimulação podem produzir contração muscular artificial e consequentemente aumentar a pressão intra-abdominal e produzir repercussóes, como o aumento do pico de fluxo expiratório, melhor função muscular e pulmonar em indivíduos com lesões medulares crônicas e agudas, com diferentes níveis de lesão (do terceiro nível cervical (C3) ao décimo segundo torácico (T12) e classificação (completa e incompleta).

Alguns estudos mostram eletroestimulação de superfície em pacientes com lesão medular espinal em ventilação mecânica invasiva, mas não se trata do objetivo desta revisão. No entanto, em pacientes com lesão medular espinal, hemodinamicamente estáveis, entre os níveis cervicais a torácicos, mesmo livres de ventilação invasiva ou não-invasiva é recomendada a aplicação imediata de estimulação elétrica de superfície nos músculos abdominais expiratórios. Esta recomendação é respaldada pela presença de precoce sintomatologia de disfunção muscular expiratória em detrimento da paralisia muscular respiratória. ${ }^{(17-27)}$

O tempo de intervenção pode variar conforme o desempenho muscular respiratório do indivíduo com lesão medular, sendo indispensável a avaliação longitudinal da função muscular respiratória e dos sintomas clínicos relacionados às infecçóes respiratórias. Nesse sentindo, existem propostas de intervenção e acompanhamento no período de até 6 meses, neste acompanhamento, verifica-se, além de melhores níveis nas variáveis de função muscular respiratória e pulmonar, como o pico de fluxo expiratório, a redução na frequência de infecçôes respiratórias nos pacientes submetidos à estimulação elétrica nos músculos expiratórios. ${ }^{(21,26)}$

A aplicação da eletroestimulação não invasiva para fins de tratamento da função muscular respiratória é recomendada por meio de eletrodos de superfície localizados em músculos expiratórios e acessórios, como o reto e oblíquo abdominal, dentre outros, como descritos no quadro 1 . Foram usados eletrodos com dife- 
rentes formatos (redondos, retangulares e quadrados), dimensão e número, sendo a localização determinada com maior precisão por eletrodiagnóstico dos pontos motores. Já o meio utilizado para contato entre o eletrodo e a pele, e consequente para condução de corrente elétrica do equipamento para fibra neuromotora foi o gel condutor à base de água. E apesar do fluxo da corrente ser preferencialmente em trajetos com menor impedância, não foram relatados preparados antecedentes à colocação dos eletrodos, como tricotomia e assepsia local. ${ }^{(17-27)}$

O posicionamento dos pacientes durante os processos de avaliação e intervenção eletroterapêutica revelou a preferência entre as posiçóes supinas e/ou sentadas, o que pode estar relacionado a diversos fatores, como classificação da lesão medular, função pulmonar, nível de independência funcional e autonomia do paciente. Além disso, outros parâmetros apresentam maior relevância e notoriedade quando se trata de modalidades eletroterapêuticas, como no caso da dosimetria aplicada para assistência muscular respiratória. ${ }^{(17-27)}$

Nos parâmetros de dosimetria identificados para promover contração funcional para função respiratória houve predominância de frequências de 30 a $50 \mathrm{~Hz}$, geralmente associadas a $300 \mu$ s de largura de pulso e amplitude de corrente de até 350 $\mathrm{mA}$. Correntes com estas características são capazes de estimular fibras profundas e de maior limiar. A amplitude da corrente foi estimada pela contraçáo visível ou pela sensibilidade de cada paciente. ${ }^{(17-27)} \mathrm{A}$ modulação da frequência está relacionada ao recrutamento da fibra muscular, e consequentemente ao condicionamento da contração, sendo em faixas superiores a $30 \mathrm{~Hz}$ possível gerar estímulos nas fibras de contração rápida e lenta. ${ }^{(34,35)}$

Outras variáveis relevantes foram frequentemente omitidas nos estudos avaliados. Alguns exemplos são a forma de onda de corrente e o tempo de subida, este definido como o intervalo necessário para se obter a amplitude final dos pulsos de corrente. Apenas um estudo relatou este tempo (no caso, de 0,5 segundo), juntamente com os tempos de on (contração) e off(repouso), identificados respectivamente como 4 segundos cada. ${ }^{(21)}$

A aplicaçáo de faixas de frequência entre $30 \mathrm{~Hz}$ e $50 \mathrm{~Hz}$ associada à largura de pulso média de $300 \mu \mathrm{s}$ demonstrou que essa experiência obteve aumento do pico de fluxo expiratório e menor ocorrência de infecçóes pulmonares em relação ao grupo tratado sem eletroestimulação. ${ }^{(17-27)}$ Esses parâmetros podem estar associados ao condicionamento de contraçáo muscular respiratória intermitente que promove pressão aumento da pressão intra-abdominal semelhante ao mecanismo fisiológico de respiração e tosse.

É imprescindível esclarecer que os estudos incluídos apresentam fragilidades metodológicas, sendo o desempenho mensurado apresentado nas tabelas 1 e 2, que correspondem aos estudos observacionais e ensaios clínicos aleatórios, respectivamente. Cabe ressaltar que nenhum dos estudos realizou cálculo amostral, critério preconizado para pesquisas bem conduzidas. Os estudos também não mencionaram a fonte de financiamento e as medidas adotadas para evitar potenciais vieses. Estes pontos fragilizam conclusóes precisas a respeito da intervenção fisioterapêutica por meio de eletroestimulação de superfície para assistência respiratória em pessoas com lesão medular. Neste sentindo, novos ensaios clínicos randomizados, seguindo recomendaçóes de delineamento apropriado, deverão serem realizados para melhor elucidação dos efeitos da eletroestimulação de superfície sobre a função pulmonar e em relação às complicaçóes respiratórias nos pacientes com lesão medular.

\section{Conclusão}

As evidências avaliadas revelam que a estimulação elétrica de superfície é uma alternativa para intervenção fisioterapêutica capaz de alterar e melhorar o desempenho de variáveis da função muscular respiratória, pulmonar e de tosse em pessoas com lesão medular espinal. No entanto, apesar dos resultados majoritariamente positivos, não se pode afirmar que eletroestimulação de superfície na função respiratória do contingente acompanhado apresenta resultados superiores a condutas fisioterapêuticas tradicionais, como a assistência fisioterapêutica manual para condicionamento da pressão intra-abdominal. Diante dos achados, os autores encorajam novas pesquisas em torno do tema. 


\section{Agradecimentos}

Os autores agradecem a bolsa de fomento da Coordenação de Aperfeiçoamento de Pessoal de Nível Superior (CAPES) e ao Conselho Nacional de Desenvolvimento Cientifico e Tecnológico (CNPq) pela bolsa produtividade.

\section{Colaborações}

Macedo FS, Paz CCSC, Rocha AF, Miosso CJ, Carvalho $\mathrm{HB}$ e Mateus SRM contribuíram com a concepção e desenho do estudo, análise e interpretação de dados para o trabalho, redação do artigo, revisão crítica relevante do conteúdo intelectual e aprovação final da versão a ser publicada.

\section{Referências}

1. Brasil. Ministério da Saúde. Secretaria de Atenção à Saúde. Diretrizes de Atenção à Pessoa com Lesão Medular. Brasilia (DF): Ministério da Saúde; 2015.

2. National Spinal Cord Injury Statistical Center. Complete public version of the 2016 annual statistical report for the spinal cord injury model systems. Birmingham: National Spinal Cord Injury Statistical Center; 2016.

3. Singh A, Tetreault L, Kalsi-Ryan S, Nouri A, Fehlings MG. Global Prevalence and incidence of traumatic spinal cord injury. Clin Epidemiol. 2014; 6(2014):309-31.

4. Lee SY, Cho KJ. A study on the cough augmentation using a belt-driven assistive. IEEE Int Conf Rehabil Robot. 2014; 6(2014):309-31.

5. Berlowitz DJ, Wadsworth B, Ross J. Respiratory problems and management in the patient with spinal cord injury. Breathe. 2016; 12(4):328-40

6. Craven C, Hitzig SL, Mittmann N. Impact of impairment and secondary health conditions on health preference among Canadians with chronic spinal cord injury. J Spinal Cord Med. 2012; 35(5):361-70.

7. Vazquez RG, Sedes PR, Farina MM, Marques AM, Velasco MEF. Respiratory management in the patient with spinal cord injury. Biomed Res Int. 2013; 2013(2013):1-12.

8. Bach JR. Noninvasive respiratory management and diaphragm and electrophrenic pacing in neuromuscular disease and spinal cord injury. Muscle Nerve. 2013[cited 2014; 47(2):297-305.

9. Bach JR. Noninvasive respiratory management of high level spinal cord injury. J Spinal Cord Med. 2012; 35(2):72-80.

10. Postma K, Haisma JA, Hopman MTE, Bergen MP, Stam HJ, Bussmann JB. Resistive inspiratory muscle training in people with spinal cord injury during inpatient rehabilitation: a randomized controlled trial. Phys Ther. 2014; 94(12):1709-1

11. Ibitoye MO, Estigoni EH, Hamzaid NA, Wahab AKA, Davis GM. The effectiveness of FES-Evoked EMG potentials to assess muscle force and fatigue in individuals with spinal cord injury. Sensors (Basel). 2014; 14(7):12599-22.
12. McLachlan AJ, McLean AN, Allan DB, Gollee H. Changes in pulmonary function measures following a passive abdominal functional electrical stimulation training program. J Spinal Cord Med. 2013; 36(2):97103.

13. levins A, Moritz CT. Therapeutic Stimulation for Restoration of Function After Spinal Cord Injury]. Physiology. 2017; 32(5):391-398.

14. Moher D, Shamseer L, Clarke M, Ghersi D, Liberati A, Petticrew M, Shekelle P, Stewart LA, PRISMA-P Group. Preferred reporting items for systematic reviewand meta-analysis protocols (PRISMA-P) 2015 statement. BioMed Central. 2015; 4(1):1-9.

15. Physiotherapy Evidence Database. [Escala PEDro]; Physiotherapy Evidence Database. [Internet]. 2016[cited 2014 Mar 10]. Avaliable from: https://www.pedro.org.au/wp-content/uploads/PEDro_scale_ portuguese(brasil).pdf.

16. Malta M, Cardoso LO, Bastos FI, Magnanini MM, Silva MF. Iniciativa STROBE: subsídios para a comunicação de estudos observacionais. Rev Saúde Pública. 2010; 44(3):559-65.

17. Linder SH. Functional electrical stimulation to enhance cough in quadriplegia functional electrical stimulation enhance cough in quadriplegia. Chest. 1993; 103(1):166-9.

18. Jaeger RJ, Turba RM, Yarkony GM, Roth EJ. Cough in spinal cord injured patients: comparison of three methods to produce cough. Arch Phys Med Rehabil. 1993; 72(12):1358-61.

19. Sorli J, Kandare F, Jaeger RJ, Stanic U. Ventilatory assistance using electrical stimulation of abdominal muscles. IEEE Trans Rehabil Eng. 1996; 4(1):1-6.

20. Zupan A, Savrin R, Erjavec T, Kralj A, Karcnik T, Skorjanc T, Benko H, Obreza P. Effects of respiratory muscle training and electrical stimulation of abdominal muscles on respiratory capabilities in tetraplegic patients. Spinal cord. 1997; 35(8):540-5.

21. Cheng P, Chen C, Wang C, Chung C. Effect of neuromuscular electrical stimulation on cough capacity and pulmonary function in patients with acute cervical cord injury. J Rehabil Med. 2006; 38(1):32-6.

22. Gollee H, Hunt KJ, Allan DB, Fraser MH, McLean NA. A control system for automatic electrical stimulation of abdominal muscles to assist respiratory function in tetraplegia. Med Eng Phys. 2006; 29(7):799-80.

23. Spivak E, Keren O, Niv D, Levental J, Steinberg F, Barak D, et al. Electromyographic signal-activated functional electrical stimulation of abdominal muscles: the effect on pulmonary function in patients with tetraplegia. Spinal Cord. 45(7):491-5.

24. Gollee H, Hunt KJ, Allan DB, Fraser MH, McLean N. Automatic electrical stimulation of abdominal wall muscles increases tidal volume and cough peak flow in tetraplegia. Technol Health Care. 2008; 16(4):273-81.

25. Butler JE, Lim J, Gorman RB, Boswell-Ruys C, Saboisky JP, Lee BB, Gandevia SC. Posterolateral surface electrical stimulation of abdominal expiratory muscles to enhance cough in spinal cord injury. Neurorehabil Neural Repair. 2011; 25(2):158-67.

26. Mcbain RA, Boswell-Ruys CL, Lee BB, Gandevia SC, Butler JE. Abdominal muscle training can enhance cough after spinal cord injury. Neurorehabil Neural Repair. 2013; 27(9):834-43.

27. Mcbain RA, Boswell-Ruys CL, Lee BB, Gandevia SC, Butler JE. Electrical stimulation of abdominal muscles to produce cough in spinal cord injury: effect of stimulus intensity. Neurorehbil Neural Repair. 2015; 29(4):1-8.

28. Savic G, DeVivo MJ, Frankel HL, Jamous MA, Soni BM, Charlifue S. Long-term survival after traumatic spinal cord injury: a 70-year British study. Spinal Cord. 2017; 55(7):651-8. 
29. DeVivo MJ. Epidemiology of traumatic spinal cord injury: trends and future implications. Spinal Cord. 2012;50(5):365-72.

30. Weinberg DS, Hedges BZ, Belding JE, Moore TA, Vallier HA. Risk factors for pulmonary complication following fixation of spine fractures. Spine J. 2017; 17(10):1449-56.

31. Postma K, Haisma JA, De Groot S, Hopman MT, Bergen MP, Stam HJ, et al. Changes in pulmonary function during the early years after inpatient rehabilitation in persons with spinal cord injury: A prospective cohort study. Arch Phys Med Rehabil. 2013; 60(3):602-9.
32. Ho CH, Triolo RJ, Elias AL, Kilgore KL, DiMarco AF, Bogie $K$, et al. Functional electrical stimulation and spinal cord injury. Phys Med Rehab Clin North Am. 2014; 25(3):631-61.

33. Stiller K. Physiotherapy in intensive care: an updated systematic review. Chest 2013; 144(3):825-47.

34. Segers J, Hermans G, Bruyninckx F, Meyfroidt G, Langer D, Gosselink R. Feasibility of neuromuscular electrical stimulation in critically ill patients. J Crit Care. 2014; 29(6):1082-8.

35. Prentice WE. Modalidades terapêuticas para fisioterapeutas. Rio de Janeiro: MacGrawHill; 2014. 